\title{
Oxidative stress induces CHIP-mediated ubiquitination and roteasomal degradation of soluble guanylyl cyclase Sabine Meurer ${ }^{1,2}$, Tatjana Pabst ${ }^{1}$, Sylke Pioch ${ }^{1}$, Nils Opitz ${ }^{1,2}$, Peter M Schmidt ${ }^{2}$, Kristina Wagner ${ }^{1}$, Simone Matt ${ }^{1}$, Harald HHW Schmidt ${ }^{2}$ and Werner Müller-Esterl*1
}

Address: ${ }^{1}$ Institute of Biochemistry II, University of Frankfurt Medical School, Theodor-Stern-Kai 7, Frankfurt, Germany and ${ }^{2}$ Department of Pharmacology \& Centre for Vascular Health, Monash University, Clayton, Victoria, Australia

Email: Werner Müller-Esterl* - wme@biochem2.de

* Corresponding author

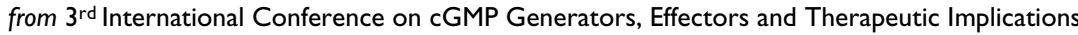

Dresden, Germany. 15-17 June 2007

Published: 25 July 2007

BMC Pharmacology 2007, 7(Suppl I):S34 doi:I0.II86/I47I-22I0-7-SI-S34

This abstract is available from: http://www.biomedcentral.com//47I-2210/7/SI/S34

(C) 2007 Meurer et al; licensee BioMed Central Ltd.

Oxidative stress attenuates the NO-cGMP pathway, e.g. in the vascular system, through scavenging of free NO radicals by superoxide $\mathrm{O}_{2}{ }^{-}$, by inactivation of soluble guanylyl cyclase (sGC) via oxidation of its central $\mathrm{Fe}^{2+}$ ion, and by down-regulation of sGC protein levels. While the former pathways are well established, the molecular mechanisms underlying the latter are still obscure. Using oxidative sGC inhibitor ODQ we demonstrate rapid down-regulation of sGC protein in mammalian cells. Coincubation with proteasomal inhibitor MG132 results in accumulation of ubiquitinated sGC whereas sGC activator BAY 58-2667 prevents ubiquitination. ODQ-induced down-regulation of sGC is mediated through selective ubiquitination of its b subunit, and BAY 58-2667 abrogates this effect. Ubiquitination of sGC-b is dramatically enhanced by E3 ligase CHIP. Our data indicate that oxidative stress promotes ubiquitination of sGC b subunit through E3 ligase CHIP, and that sGC activator 58-2667 reverts this effect, most likely through stabilization of the heme-free b subunit. Thus the deleterious effects of oxidative stress can be counter-balanced by an activator of a key enzyme of vascular homeostasis. 